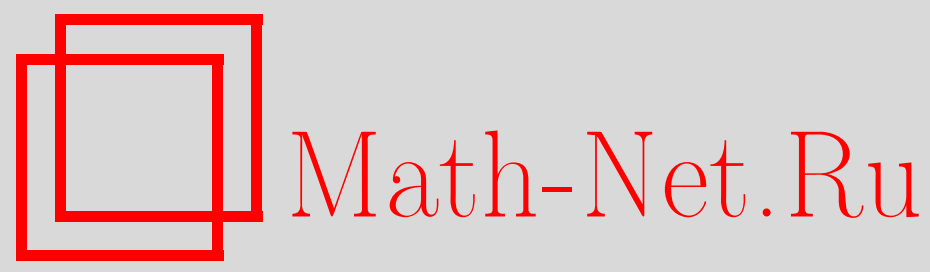

В. И. Овчинников, А. С. Титенков, Критерий родственности квазивогнутых функций, Матем. заметки, 2001, том 70, выпуск 5, 780-786

DOI: https://doi.org/10.4213/mzm789

Использование Общероссийского математического портала Math-Net.Ru подразумевает, что вы прочитали и согласны с пользовательским соглашением http://www.mathnet.ru/rus/agreement

Параметры загрузки:

IP : 3.95 .254 .165

26 апреля 2023 г., 18:12:54

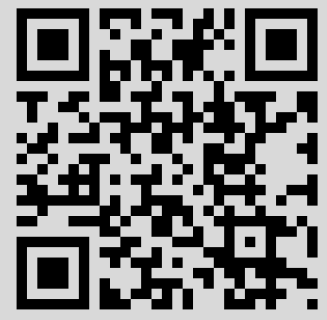




\title{
КРИТЕРИЙ РОДСТВЕННОСТИ КВАЗИВОГНУТЫХ ФУНКЦИЙ
}

\author{
В.И. Овчинников, А.С. Титенков
}

Квазивогнутые функции $\rho_{0}$ и $\rho_{1}$ принадлежат одной шкале, если существуют квазивогнутые функции $\psi_{0}$ и $\psi_{1}$ и числа $0<\theta_{0}<1,0<\theta_{1}<1$ такие, что $\rho_{0}=\psi_{0}^{1-\theta_{0}} \psi_{1}^{\theta_{0}}$, $\rho_{1}=\psi_{0}^{1-\theta_{1}} \psi_{1}^{\theta_{1}}$. В работе установлен критерий принадлежности с точностью до эквивалентности функций к одной шкале. Этот критерий получен в терминах узлов соответствующих им линейно ступенчатых функций. Оказалось, что узлы должны быть эквивалентными последовательностями.

Библиографоия: 10 названий.

Как известно, функция $\varphi(t)$ на полуоси $[0, \infty)$ называется квазивогнутой, если $\varphi(0)=0$, функция $\varphi(t)$ положительна и возрастает при $t>0$, а функция $\varphi(t) / t$ убывает при $t>0$. Любая возрастающая, положительная на $[0, \infty)$ вогнутая функция является квазивогнутой. Эти функции используются как обобщенные гладкости (см., например, [1]). Они также возникают в качестве функциональных параметров в конструкциях разного рода пространств в теории функций и теории приближений. При этом обычно эквивалентные функции оказываются взаимозаменяемыми. Положительные функции назьваются әквивалентными, если их отношение ограничено сверху и снизу положительными константами. Любая квазивогнутая функция эквивалентна возрастающей вогнутой функции. Этим пользуются в том смысле, что произвольную квазивогнутую функцию заменяют эквивалентной ей вогнутой, а ту, в свою очередь, можно заменить даже на эквивалентную ей лернеровскую гладкую функцию (см. [2]). Оказалось же, что в ряде вопросов разумнее идти в прямо противоположном направлении, т.е. заменять вогнутые гладкие функции на эквивалентные квазивогнутые “экстремально" невогнутые функции. Так возникают линейно ступенчатые функции, которые целиком определяются возрастающей последовательностью положительных чисел (узлов) на полуоси (см. [3]).

Через $\mathbb{Z}$, как обычно, обозначим множество целых чисел. Интервалом $M$ будем назьвать множество целых чисел вида $\{n \in \mathbb{Z} \mid \alpha<n<\beta\}$, где $-\infty \leqslant \alpha<\beta \leqslant \infty$.

Монотонно возрастающую последовательность $\left\{r_{n}\right\}_{n \in M}$, где $r_{n} \in[0, \infty]$, занумерованную элементами из интервала $M$, будем назьвать последовательностью узлов, если $\sup _{n \in M} r_{n}=\infty, \operatorname{anf}_{n \in M} r_{n}=0$.

ОпрЕДЕЛЕниЕ 1. Непрерьвная положительная функция $\varphi$ на $[0, \infty)$ назьвается $л и$ нейно ступенчатой функиией, если существует последовательность узлов $\left\{r_{n}\right\}_{n \in M}$ 
такая, что функция $\varphi$ постоянна при $t \in\left[r_{2 n}, r_{2 n+1}\right]$ и линейная однородная при $t \in$ $\left[r_{2 n-1}, r_{2 n}\right]$.

Один из основных результатов работы [3] - это теорема о том, что любая квазивогнутая функция эквивалентна некоторой линейно ступенчатой функции. Нетрудно заметить связь этой простой теоремы с аналогичными результатами К. И. Осколкова [4], С. Янсона [5], Ю.А. Брудного и Н.Я. Кругляка [6] о дискретизации квазивогнутых функций. Однако есть и существенные отличия, связанные с рассмотрением как четных, так и нечетных узлов (нечетные узлы ранее либо игнорировались, либо дискретизация строилась без них). Привлечение нечетных узлов оказалось очень продуктивным при вычислении индексов растяжения квазивогнутых функций, а также при решении некоторых задач теории интерполяции линейных операторов (см. [7], [8]). В данной работе с помощью линейно ступенчатых функций найдено новое описание одной классификации квазивогнутых функций, обнаруженной недавно Ю. А. Брудным и А. С. Штейнбергом $[9]$.

ОПРЕДЕЛЕНИЕ 2. Говорят, что две квазивогнутые функции $\rho_{0}$ и $\rho_{1}$ принадлежат одной шкале, если существуют квазивогнутые функции $\psi_{0}$ и $\psi_{1}$ и числа $0<\theta_{0}<1$, $0<\theta_{1}<1$ такие, что $\rho_{0}=\psi_{0}^{1-\theta_{0}} \psi_{1}^{\theta_{0}}, \rho_{1}=\psi_{0}^{1-\theta_{1}} \psi_{1}^{\theta_{1}}$.

ОПРЕДЕЛЕНИЕ 3. Две квазивогнутые функции мы будем называть родственными, если они эквивалентны функциям из одной шкалы.

В работе [9] было отмечено, что понятие родственности обладает свойством транзитивности, т.е. если $f$ родственна $g$, а $g$ родственна $h$, то $f$ родственна $h$.

Тем самым возникает разбиение множества квазивогнутых функций на классы родственных. Первым шагом такой классификации, в частности, является хорошо известное выделение класса квазистепенных функций, которые оказываются родственньпи друг другу.

Интерес к этому понятию связан с тем, что в терминах родственности формулируются критерии $K$-монотонности пар пространств Янсона с функциональными параметрами (см. [9]), а также условия на функциональные параметры в теореме о реитерации для интерполяционных конструкций типа Гальярдо (см. [10]).

Поскольку любая квазивогнутая функция эквивалентна некоторой линейно ступенчатой функции, которая практически однозначно определяется своими узлами, то естественно, что понятие родственности каким-то образом выражается через узлы соответствующих линейно ступенчатых функций. Решению этого вопроса и посвящена данная работа. Оказалось, что родственность линейно ступенчатых функций непосредственно связана с эквивалентностью последовательностей их узлов.

1. Вспомогательные теоремы. Квазивогнутая функция $\varphi$ называется невырожденной, если области значений функций $\varphi(t)$ и $t / \varphi(t)$ содержат полуось $(0, \infty)$. Для линейно ступенчатой функции это значит, что последовательность ее узлов является бесконечной двусторонней последовательностью. Только такие функции для простоты мы в дальнейшем и будем рассматривать.

Узлы $\left\{r_{n}\right\}$ линейно ступенчатой функции называются $р а з$ реженным.ми, если $r_{n+1} / r_{n}$ $\geqslant q$ для некоторой константы $q>1$ при всех $n$. В работе [3] доказано, что любая невырожденная квазивогнутая функция эквивалентна линейно ступенчатой функции, узлы 
которой разрежены, причем $q>1$ можно взять любое. Поэтому мы будем рассматривать, если не оговорено иное, только такие линейно ступенчатые функции, у которых $q>2$.

ТеОрема 1. Если у линейно ступенчатых функиий $\varphi_{0}$ и $\varphi_{1}$ четные узлы совпадают, а нечетные әквивалентны, то эти функиии родственные.

Доказательство опирается на следующие леммы.

Лемма 1. Пусть $\psi_{0} и \psi_{1}-$ две линейно ступенчатые функиии, $a\left\{\nu_{n}\right\} u\left\{\omega_{n}\right\}-$ соответственно последовательности их узлов, которые разрежсены. Пусть четнье узль этих последовательностей совпадают, $\nu_{2 n}=\omega_{2 n}$, а нечетные әквивалентны. Тогда при любом $\theta \in(0,1)$ линейно ступенчатая функиия $\bar{\psi}$ с узлами $t_{n}=\nu_{n}^{1-\theta} \omega_{n}^{\theta}$ әквивалентна функиии $\psi=\psi_{0}^{1-\theta} \psi_{1}^{\theta}$.

ДокАЗАТЕЛЬСТВо. Неограничивая общности, можно полагать, что $\nu_{2 n+1} \leqslant \omega_{2 n+1}$. Обозначим на отрезках $\left[\nu_{2 n}, \nu_{2 n+1}\right]$ функцию $\psi_{0}(t)=c_{n}$. На отрезках $\left[\nu_{2 n+1}, \nu_{2 n+2}\right]$ функция $\psi_{0}(t)=k_{n} t$. Аналогично функция $\psi_{1}$ на отрезках $\left[\nu_{2 n}, \omega_{2 n+1}\right]$ постоянна, т.е. $\psi_{1}(t)=d_{n}$, где $d_{n}$ - некоторая константа. На $\left[\omega_{2 n+1}, \nu_{2 n+2}\right]$ функция $\psi_{1}(t)=m_{n} t$. Очевидно, $\nu_{2 n+1}=c_{n} / k_{n}$ и $\omega_{2 n+1}=d_{n} / m_{n}$. Нетрудно видеть, что на отрезках $\left[\nu_{2 n}, \nu_{2 n+1}\right]$ функция $\psi_{0}^{1-\theta} \psi_{1}^{\theta}=c_{n}^{1-\theta} d_{n}^{\theta}$. На отрезках $\left[\nu_{2 n+1}, \omega_{2 n+1}\right]$ функция $\psi_{0}^{1-\theta} \psi_{1}^{\theta}=\left(k_{n} t\right)^{1-\theta} d_{n}^{\theta}$, а на отрезках $\left[\omega_{2 n+1}, \nu_{2 n+2}\right]$ функция $\psi_{0}^{1-\theta} \psi_{1}^{\theta}=k_{n}^{1-\theta} m_{n}^{\theta} t$. Теперь рассмотрим на каждом отрезке $\left[\nu_{2 n}, \nu_{2 n+2}\right]$ функцию $\bar{\psi}(t)=\max \left(c_{n}^{1-\theta} d_{n}^{\theta}, k_{n}^{1-\theta} m_{n}^{\theta} t\right)$. Обозначим через $t_{2 n+1}$ абсциссу точки пересечения прямой $y=k_{n}^{1-\theta} m_{n}^{\theta} t$ и прямой $y=c_{n}^{1-\theta} d_{n}^{\theta}$. Тогда

$$
t_{2 n+1}=\left(\frac{c_{n}}{k_{n}}\right)^{1-\theta}\left(\frac{d_{n}}{m_{n}}\right)^{\theta}=\nu_{2 n+1}^{1-\theta} \omega_{2 n+1}^{\theta} .
$$

Очевидно, что функция $\bar{\psi}(t)=\max \left(c_{n}^{1-\theta} d_{n}^{\theta}, k_{n}^{1-\theta} m_{n}^{\theta} t\right)$ будет линейно ступенчатой, и ее узлы $t_{2 n}=\nu_{2 n}=\omega_{2 n}$, а $t_{2 n+1}=\nu_{2 n+1}^{1-\theta} \omega_{2 n+1}^{\theta}$ при любом $n \in \mathbb{Z}$. Покажем, что функция $\psi$ эквивалентна $\bar{\psi}$. Действительно, при любом $n$ и $t \in\left[\nu_{2 n}, \nu_{2 n+2}\right]$

$$
\begin{aligned}
1 & \leqslant \frac{\psi(t)}{\bar{\psi}(t)} \leqslant \frac{\psi\left(t_{2 n+1}\right)}{\bar{\psi}\left(t_{2 n+1}\right)}=\frac{k_{n}^{1-\theta} t_{2 n+1}^{1-\theta} d_{n}^{\theta}}{c_{n}^{1-\theta} d_{n}^{\theta}}=\left(\frac{k_{n}}{c_{n}}\right)^{1-\theta}\left(\left(\frac{c_{n}}{k_{n}}\right)^{1-\theta}\left(\frac{d_{n}}{m_{n}}\right)^{\theta}\right)^{1-\theta} \\
& =\left(\left(\frac{c_{n}}{k_{n}}\right)^{-\theta}\left(\frac{d_{n}}{m_{n}}\right)^{\theta}\right)^{1-\theta}=\left(\frac{\omega_{2 n+1}}{\nu_{2 n+1}}\right)^{(1-\theta) \theta} \leqslant M^{(1-\theta) \theta}
\end{aligned}
$$

где $M-$ константа в условии $\omega_{2 n+1} \leqslant M \nu_{2 n+1}$. Лемма доказана.

Лемма 2. Пусть $f_{0} u f_{1}$ - две линейно ступенчатые функиии, $a\left\{\gamma_{n}\right\} u\left\{\tau_{n}\right\}-$ соответственно последовательности их узлов, и у этих функиий узль разрехены. Пусть четные узль этих последовательностей совпадают, $\gamma_{2 n}=\tau_{2 n}$, анечетные узлы әквивалентны $и \gamma_{2 n+1} \leqslant \tau_{2 n+1}$ при любом $n \in \mathbb{Z}$. Тогда функиии $f_{0}$ u $f_{1}$ родственные. 
ДоКАЗАТЕЛЬСТВО. Построим две линейно ступенчатые функции $\psi_{0}$ и $\psi_{1}$, у которых четные узлы совпадают с соответствующими четньми узлами функций $f_{0}$ и $f_{1}$, т.е. $\nu_{2 n}=\omega_{2 n}=\gamma_{2 n}=\tau_{2 n}$, а нечетные узлы $\nu_{2 n+1}$ и $\omega_{2 n+1}$ эквивалентны и $\nu_{2 n+1}^{1-\theta_{0}} \omega_{2 n+1}^{\theta_{0}}=$ $\gamma_{2 n+1}$, а $\nu_{2 n+1}^{1-\theta_{1}} \omega_{2 n+1}^{\theta_{1}}=\tau_{2 n+1}$ для некоторых $0<\theta_{0}<\theta_{1}<1$. Последние два равенства подразумевают, что $\nu_{2 n+1} \leqslant \gamma_{2 n+1} \leqslant \tau_{2 n+1} \leqslant \omega_{2 n+1}$ при всех $n$. Покажем, как найти нечетные узлы функций $\psi_{0}$ и $\psi_{1}$ по данным узлам линейно ступенчатых функций $f_{0}$ и $f_{1}$. За счет выбора начальных условий, т.е. узлов $\gamma_{1}, \tau_{1}, \nu_{1}$ и $\omega_{1}$, из уравнений $\nu_{1}^{1-\theta_{0}} \omega_{1}^{\theta_{0}}=\gamma_{1}$ и $\nu_{1}^{1-\theta_{1}} \omega_{1}^{\theta_{1}}=\tau_{1}$ находим $\theta_{0}$ и $\theta_{1}$. При фиксированных $\theta_{0}$ и $\theta_{1}$ из двух уравнений $\nu_{n}^{1-\theta_{0}} \omega_{n}^{\theta_{0}}=\gamma_{n}$ и $\nu_{n}^{1-\theta_{1}} \omega_{n}^{\theta_{1}}=\tau_{n}$ получим формулы для узлов $\nu_{n}$ и $\omega_{n}$, т.е.

$$
\nu_{n}=\gamma_{n}^{\theta_{1} /\left(\theta_{1}-\theta_{0}\right)} \tau_{n}^{-\theta_{0} /\left(\theta_{1}-\theta_{0}\right)}, \quad \omega_{n}=\gamma_{n}^{-\left(1-\theta_{1}\right) /\left(\theta_{1}-\theta_{0}\right)} \tau_{n}^{\left(1-\theta_{0}\right) /\left(\theta_{1}-\theta_{0}\right)}
$$

Для завершения доказательства нужно подобрать теперь $0<\theta_{0}<\theta_{1}<1$ так, чтобы полученные последовательности $\left\{\nu_{n}\right\}$ и $\left\{\omega_{n}\right\}$ были разрежены, т.е. $\nu_{n+1} / \nu_{n} \geqslant \beta>2$ и $\omega_{n+1} / \omega_{n} \geqslant \beta>2$. В самом деле,

$$
\frac{\omega_{2 n+1}}{\omega_{2 n}}=\frac{\gamma_{2 n+1}^{-\left(1-\theta_{1}\right) /\left(\theta_{1}-\theta_{0}\right)} \tau_{2 n+1}^{\left(1-\theta_{0}\right) /\left(\theta_{1}-\theta_{0}\right)}}{\gamma_{2 n}}=\left(\frac{\tau_{2 n+1}}{\gamma_{2 n+1}}\right)^{\left(1-\theta_{0}\right) /\left(\theta_{1}-\theta_{0}\right)} \frac{\gamma_{2 n+1}}{\gamma_{2 n}} \geqslant \beta>2,
$$

поскольку $\gamma_{2 n+1} \leqslant \tau_{2 n+1} \leqslant k \gamma_{2 n+1}$ и $\gamma_{2 n+1} / \gamma_{2 n} \geqslant \beta>2$ по условию леммы.

Также имеем, что

$$
\frac{\nu_{2 n}}{\nu_{2 n-1}}=\frac{\nu_{2 n}}{\left(\gamma_{2 n-1}^{\theta_{1}} \tau_{2 n-1}^{-\theta_{0}}\right)^{1 /\left(\theta_{1}-\theta_{0}\right)}}=\frac{\tau_{2 n}}{\tau_{2 n-1}}\left(\frac{\tau_{2 n-1}}{\gamma_{2 n-1}}\right)^{\theta_{1} /\left(\theta_{1}-\theta_{0}\right)} \geqslant \beta>2 .
$$

Теперь найдем отношение

$$
\begin{aligned}
\frac{\nu_{2 n+1}}{\nu_{2 n}} & =\frac{\gamma_{2 n+1}^{\theta_{1} /\left(\theta_{1}-\theta_{0}\right)} \tau_{2 n+1}^{-\theta_{0} /\left(\theta_{1}-\theta_{0}\right)}}{\gamma_{2 n}}=\frac{\gamma_{2 n+1}}{\gamma_{2 n}}\left(\frac{\gamma_{2 n+1}}{\tau_{2 n+1}}\right)^{\theta_{0} /\left(\theta_{1}-\theta_{0}\right)} \\
& \geqslant \beta\left(\frac{1}{k}\right)^{\theta_{0} /\left(\theta_{1}-\theta_{0}\right)} \geqslant \beta-\varepsilon>2
\end{aligned}
$$

где $\varepsilon$ достаточно мало. Здесь мы используем разреженность узлов и неравенство $1 \geqslant$ $\gamma_{2 n+1} / \tau_{2 n+1} \geqslant k^{-1}$, а также то, что при $\theta_{0} \rightarrow 0$ выражение $(1 / k)^{\theta_{0} /\left(\theta_{1}-\theta_{0}\right)} \rightarrow 1$. Аналогично,

$$
\frac{\omega_{2 n}}{\omega_{2 n-1}}=\frac{\omega_{2 n}}{\left.\left(\gamma_{2 n-1}^{-1+\theta_{1}} \tau_{2 n-1}^{1-\theta_{0}}\right)^{1 /\left(\theta_{1}-\theta_{0}\right)}\right)}=\frac{\tau_{2 n}}{\tau_{2 n-1}}\left(\frac{\tau_{2 n-1}}{\gamma_{2 n-1}}\right)^{-\left(1-\theta_{1}\right) /\left(\theta_{1}-\theta_{0}\right)} \geqslant \beta-\varepsilon>2,
$$

поскольку при $\theta_{1} \rightarrow 0$ имеем $\left(\tau_{2 n-1} / \gamma_{2 n-1}\right)^{\left(\theta_{1}-1\right) /\left(\theta_{1}-\theta_{0}\right)} \rightarrow 1$.

Из леммы 1 сразу следует, что функция $f_{0}$ эквивалентна функции $\psi_{0}^{1-\theta_{0}} \psi_{1}^{\theta_{0}}$, а функция $f_{1}$ эквивалентна $\psi_{0}^{1-\theta_{1}} \psi_{1}^{\theta_{1}}$. Поэтому функция $f_{0}$ и $f_{1}$ будут родственными. Лемма доказана. 
ДокАЗАТЕЛЬСТВо ТЕОРЕМЫ 1. Обозначим через $\left\{r_{n}\right\}$ и $\left\{u_{n}\right\}$ последовательности узлов соответственно для функций $\varphi_{0}$ и $\varphi_{1}$. Введем в рассмотрение линейно ступенчатую функцию $\widetilde{\psi}$, у которой четные узлы $c_{2 n}=r_{2 n}=u_{2 n}$, а нечетные $c_{2 n+1}=\max \left(r_{2 n+1}\right.$, $\left.u_{2 n+1}\right)$. Очевидно, что $r_{2 n+1} \leqslant c_{2 n+1}$ и узлы $c_{2 n+1}$ эквивалентны узлам $r_{2 n+1}$. Также $c_{2 n+1} \geqslant u_{2 n+1}$ и узлы $c_{2 n+1}$ эквивалентны узлам $u_{2 n+1}$. Поэтому сразу из леммы 2 следует, что функции $\widetilde{\psi}$ и $\varphi_{0}$ родственные и функции $\widetilde{\psi}$ и $\varphi_{1}$ родственные. Следовательно, функции $\varphi_{0}$ и $\varphi_{1}$ будут родственными. Теорема доказана.

ТеОрема 2. Если у линейно ступенчатых функиий $\varphi_{0}$ и $\varphi_{1}$ нечетные узлы совпадают, а четные узлы эквивалентны, то эти функиии родственные.

ДокАЗАТЕЛьСтво. Этот результат следует из теоремы 1 , если применить ее к линейно ступенчатым функциям $\varphi_{0}^{*}(t)=1 / \varphi_{0}(1 / t)$ и $\varphi_{1}^{*}(t)=1 / \varphi_{1}(1 / t)$, у которых четные узлы совпадают, а нечетные эквивалентны. В самом деле, пусть $\left\{r_{n}^{*}\right\}$ и $\left\{u_{n}^{*}\right\}-$ последовательности узлов соответственно для функций $\varphi_{0}^{*}$ и $\varphi_{1}^{*}$. Заметим, что узлы функций $\varphi_{0}^{*}$ и $\varphi_{0}$ связаны формулой $r_{-n}^{*}=1 / r_{n+1}$. Узлы $\varphi_{1}$ и $\varphi_{1}^{*}$ связаны также формулой $u_{-n}^{*}=1 / u_{n+1}$. Теорема доказана.

\section{2. Основная теорема.}

ТЕОрема 3. Невырожсденые квазивогнутые функиии $\rho_{0}$ и $\rho_{1}$ родственны тогда и только тогда, когда мохно построить әквивалентные им линейно ступенчатые функиии $\varphi_{0}$ и $\varphi_{1}$, у которых последовательности узлов әквивалентны.

ДокАЗАТЕЛЬСТвО. Необходимость. Пусть квазивогнутые функции $\rho_{0}$ и $\rho_{1}$ родственные. Согласно утверждению, доказанному Брудным и Штейнбергом (см. [9]), эти функции имеют общую "балансировочную” последовательность $\left\{t_{k}\right\}$. Одним из свойств этой последовательности является то, что функция $\rho_{j}(t)$ эквивалентна функции

$$
\varphi_{j}(t)=\sup _{k} \rho_{j}\left(t_{k}\right) \min \left(1, \frac{t}{t_{k}}\right), \quad j=0,1 .
$$

Легко видеть, что функции $\varphi_{0}$ и $\varphi_{1}$ будут линейно ступенчатыми. Обозначим соответственно через $u_{n}$ и $r_{n}$ узлы этих функций. Общая “балансировочная" последовательность является последовательностью четных узлов для каждой из этих функций, т.e. $t_{k}=u_{2 k}=r_{2 k}$.

Поскольку функции $\varphi_{0}$ и $\varphi_{1}$ с точностью до эквивалентности принадлежат одной шкале, то для некоторого $q \geqslant 2$ должны выполняться следующие импликации (см. [9]):

$$
\begin{aligned}
\frac{\varphi_{j}(t)}{\varphi_{j}(s)} \leqslant 2 \Longrightarrow \frac{\varphi_{1-j}(t)}{\varphi_{1-j}(s)} \leqslant q, & j=0,1, \\
\frac{t \varphi_{j}(s)}{\varphi_{j}(t) s} \leqslant 2 \Longrightarrow \frac{t \varphi_{1-j}(s)}{\varphi_{1-j}(t) s} \leqslant q, & j=0,1,
\end{aligned}
$$

где $0<s<t<\infty$.

Если $u_{2 n+1} \in\left[r_{2 n+1}, r_{2 n+2}\right]$, то запишем первую импликацию для $j=1$, взяв $s$ началом, а $t$ концом отрезка постоянства $\left[r_{2 n}, u_{2 n+1}\right]$ функции $\varphi_{1}$ :

$$
\frac{\varphi_{1}\left(u_{2 n+1}\right)}{\varphi_{1}\left(r_{2 n}\right)}=1 \leqslant 2 \Longrightarrow \frac{\varphi_{0}\left(u_{2 n+1}\right)}{\varphi_{0}\left(r_{2 n}\right)}=\frac{\varphi_{0}\left(u_{2 n+1}\right)}{\varphi_{0}\left(r_{2 n+1}\right)}=\frac{u_{2 n+1}}{r_{2 n+1}} \leqslant q .
$$


Если же $r_{2 n+1} \in\left[u_{2 n+1}, r_{2 n+2}\right]$, то записьваем эту импликацию для $j=0$, взяв $s$ началом и $t$ концом отрезка постоянства $\left[r_{2 n}, r_{2 n+1}\right]$ функции $\varphi_{0}$ :

$$
\frac{\varphi_{0}\left(r_{2 n+1}\right)}{\varphi_{0}\left(r_{2 n}\right)}=1 \leqslant 2 \Longrightarrow \frac{\varphi_{1}\left(r_{2 n+1}\right)}{\varphi_{1}\left(r_{2 n}\right)}=\frac{\varphi_{1}\left(r_{2 n+1}\right)}{\varphi_{1}\left(u_{2 n+1}\right)}=\frac{r_{2 n+1}}{u_{2 n+1}} \leqslant q .
$$

Поэтому в зависимости от расположения нечетных узлов выполняется неравенство $r_{2 n+1} \leqslant u_{2 n+1} \leqslant q r_{2 n+1}$ или $u_{2 n+1} \leqslant r_{2 n+1} \leqslant q u_{2 n+1}$ при любом $n$. Это значит, что нечетные узлы функций $\varphi_{0}$ и $\varphi_{1}$ эквивалентны.

Таким образом, линейно ступенчатые функции $\varphi_{0}$ и $\varphi_{1}$ эквивалентны квазивогнутым функциям $\rho_{0}$ и $\rho_{1}$, и четные узлы этих функций совпадают, а нечетные эквивалентны.

Достаточность. Рассмотрим две линейно ступенчатые функции $\varphi_{0}$ и $\varphi_{1}$. Пусть последовательности узлов $\left\{r_{n}\right\}$ и $\left\{u_{n}\right\}$ этих функций эквивалентны, т.е. $h \leqslant u_{n} / r_{n} \leqslant H$ при всех $n \in \mathbb{Z}$. Докажем, что функции $\varphi_{0}$ и $\varphi_{1}$ родственные.

Рассмотрим семейство линейно ступенчатых функций, порожденных узлами $\omega_{n}(\alpha)=$ $r_{n}^{1-\alpha} u_{n}^{\alpha}$. Эти узлы разрежены при каждом $\alpha \in(0,1)$, поскольку мы предполагаем, что у исходных функций узлы таковы. Найдем отношение

$$
\frac{\omega_{n}\left(\alpha_{1}\right)}{\omega_{n}\left(\alpha_{0}\right)}=\frac{r_{n}^{1-\alpha_{1}} u_{n}^{\alpha_{1}}}{r_{n}^{1-\alpha_{0}} u_{n}^{\alpha_{0}}}=\left(\frac{u_{n}}{r_{n}}\right)^{\alpha_{1}-\alpha_{0}} .
$$

Поскольку узлы эквивалентны, то при $\alpha_{1}>\alpha_{0}$

$$
h^{\alpha_{1}-\alpha_{0}} \leqslant \frac{\omega_{n}\left(\alpha_{1}\right)}{\omega_{n}\left(\alpha_{0}\right)} \leqslant H^{\alpha_{1}-\alpha_{0}} .
$$

Таким образом, если параметры $\alpha_{0}$ и $\alpha_{1}$ близки, то константы эквивалентности между узлами $\omega_{n}\left(\alpha_{1}\right)$ и $\omega_{n}\left(\alpha_{0}\right)$ как угодно близки к единице. Поэтому при доказательстве мы можем считать, что константы эквивалентности между узлами исходных функций $\varphi_{0}$ и $\varphi_{1}$ как угодно близки к единице. В самом деле, если это не так, то разобьем отрезок $[0,1]$, где меняется параметр $\alpha$, на равные мелкие части так, чтобы в соседних точках разбиения константы эквивалентности узлов были близки к единице. Тогда функции, порожденные узлами $\omega_{n}(\alpha)$ с соседними $\alpha$, родственны. В силу транзитивности родственньми будут и функции, порожденные узлами $\omega_{n}(0)=r_{n}$ и $\omega_{n}(1)=u_{n}$.

Итак, пусть узлы $r_{n}$ и $u_{n}$ эквивалентны, и константы эквивалентности близки к единище, т.е. $1-\varepsilon \leqslant u_{n} / r_{n} \leqslant 1+\varepsilon$, где $\varepsilon$ достаточно мало. Напомним также, что узлы функций $\varphi_{0}$ и $\varphi_{1}$ разрежены, т.е. $r_{n+1} / r_{n} \geqslant \beta>2$ и $u_{n+1} / u_{n} \geqslant \beta>2$. Рассмотрим последовательность $\left\{c_{n}\right\}$ такую, что $c_{n}=r_{n}$ при $n=2 k$ и $c_{n}=u_{n}$ при $n=2 k+1$. Выберем $\varepsilon$ так, чтобы последовательность $\left\{c_{n}\right\}$ была разрежена. Последовательности $\left\{c_{n}\right\}$ и $\left\{r_{n}\right\}$ имеют одинаковые четные узлы, а их нечетные узлы эквивалентны. Поэтому соответствующие им линейно ступенчатые функции в силу теоремы 1 будут родственные. Последовательности $\left\{c_{n}\right\}$ и $\left\{u_{n}\right\}$ имеют одинаковые нечетные узлы, а их четные узлы эквивалентны. Значит, линейно ступенчатые функции, порожденные этими последовательностями узлов, в силу теоремы 2 будут родственные. Поэтому в силу транзитивности наши функции $\varphi_{0}$ и $\varphi_{1}$ будут родственны. Теорема доказана. 
ЗАмЕчАниЕ. Одним из прямых приложений доказанной теоремы является новое доказательство результата Брудного и Штейнберга об условиях $K$-монотонности пар пространств Янсона. Напомним, что они установили, что пара пространств $\left\{\bar{X}_{\rho_{0}}, p_{0}\right.$, $\left.\bar{X}_{\rho_{1}, p_{1}}\right\}$ является $K$-монотонной для любой исходной пары $\bar{X}$ тогда и только тогда, когда функции $\rho_{0}$ и $\rho_{1}$ являются родственными. Здесь наиболее громоздкой является доказательство необходимости этого условия. Новое доказательство получается, если применить найденное в [8] описание пространств $\left(l_{q_{0}}, l_{q_{1}}\left(2^{-n}\right)\right)_{\rho, p}$ как весовых пар пространств со смешанной $L_{p}$-нормой, построенной на основе разбиения множества индексов координат. При этом если последовательности узлов функций $\rho_{0}$ и $\rho_{1}$ неэквивалентны, то в паре $\left\{\left(l_{q_{0}}, l_{q_{1}}\left(2^{-n}\right)\right)_{\rho_{0}, p_{0}},\left(l_{q_{0}}, l_{q_{1}}\left(2^{-n}\right)\right)_{\rho_{1}, p_{1}}\right\}$ возникают дополняемые подпары вида $\left\{l_{p_{0}}\left(l_{q_{0}}\right), l_{p_{1}}\left(l_{q_{1}}\right)\right\}$, которые, как известно, не являются $K$-монотонными, если $q_{0} \neq q_{1}$ и $p_{0} \neq q_{0}$ или $p_{1} \neq q_{1}$. То есть пара $\left\{\left(l_{q_{0}}, l_{q_{1}}\left(2^{-n}\right)\right)_{\rho_{0}, p_{0}},\left(l_{q_{0}}, l_{q_{1}}\left(2^{-n}\right)\right)_{\rho_{1}, p_{1}}\right\}$ не будет $K$-монотонной.

\section{СПИСОК ЦИТИРОВАННОЙ ЛИТЕРАТУРЫ}

[1] Лизоркин П.И. Пространства обобщенной гладкости // Х. Трибель. Теория функциональных пространств. М.: Мир, 1986. С. 381-415.

[2] Овчинников В.И. Интерполяционные теоремы, вытекающие из неравенства Гротендика // Функцион. анализ и его прилож. 1976. Т. 10. № 4. С. 45-54.

[3] Титенков А.С. О показателях растяжения квазивогнутых функций // Математические науки и информационные образовательные технологии. Курск, 1997. С. 39-46.

[4] Осколков К. И. Аппроксимативные свойства суммируемых функций на множествах полной меры // Матем. сб. 1977. Т. 32. С. 480-517.

[5] Janson S. Minimal and maximal method of interpolation // J. Func. Anal. 1981. V. 44. P. 50-73.

[6] Brudnyi Ju. A., Krugliak N. Ja. Interpolation Functors and Interpolation Spaces, I. Amsterdam: North Holland, 1991.

[7] Титенков А.С. Показатели растяжения линейно ступенчатой функции. Деп. в ВИНИТИ 25.11.98, № 3478-В98. М.: ВИНИТИ, 1998.

[8] Овчинников В.И., Титенков А.С.Конструкция С. Янсона для пар пространств $L_{p} / /$ Тр. матем. факультета. № 3. Воронеж, 1998. С. 52-56.

[9] Brudnyi Ju.A., Shteinberg A. Calderon couples of Lipschitz spaces // J. Func. Anal. 1995. V. 131. P. 459-498.

[10] Ovchinnikov V. I. On reiteration theorems // Amer. Math. Soc. Transl. (2). 1998. V. 184. P. 185-198.

(В.И. Овчинников) Воронежский государственный университет 